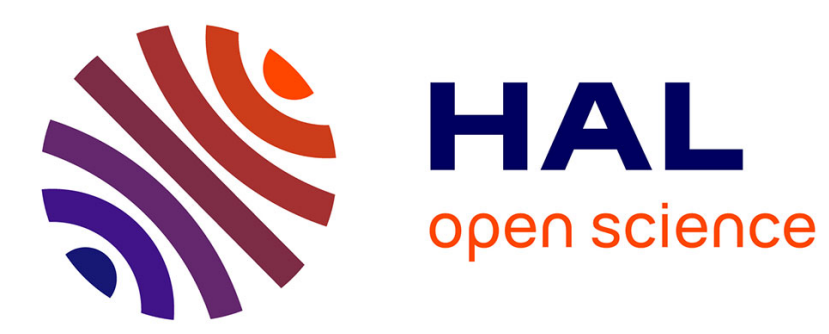

\title{
The square Thue-Morse tiling for photonic application
} Luigi Moretti, Vito Mocella

\section{To cite this version:}

Luigi Moretti, Vito Mocella. The square Thue-Morse tiling for photonic application. Philosophical Magazine, 2008, 88 (13-15), pp.2275-2284. 10.1080/14786430802047076 . hal-00513884

\section{HAL Id: hal-00513884 \\ https://hal.science/hal-00513884}

Submitted on 1 Sep 2010

HAL is a multi-disciplinary open access archive for the deposit and dissemination of scientific research documents, whether they are published or not. The documents may come from teaching and research institutions in France or abroad, or from public or private research centers.
L'archive ouverte pluridisciplinaire HAL, est destinée au dépôt et à la diffusion de documents scientifiques de niveau recherche, publiés ou non, émanant des établissements d'enseignement et de recherche français ou étrangers, des laboratoires publics ou privés. 


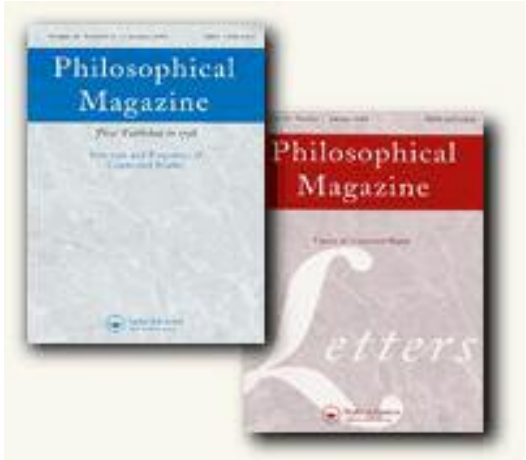

The square Thue-Morse tiling for photonic application

\begin{tabular}{|r|l|}
\hline Journal: & Philosophical Magazine \& Philosophical Magazine Letters \\
\hline Manuscript ID: & TPHM-07-Oct-0289.R1 \\
\hline Journal Selection: & Philosophical Magazine \\
\hline Date Submitted by the \\
Author: & $25-J a n-2008$ \\
\hline Complete List of Authors: & $\begin{array}{l}\text { Moretti, Luigi; University, DIMET; IMM-CNR, Dept of Naples } \\
\text { Mocella, Vito; IMM-CNR, dept of Naples }\end{array}$ \\
\hline Keywords: & optical properties, optics, quasicrystals \\
\hline Keywords (user supplied): & optical properties, optics, quasicrystals \\
\hline &
\end{tabular}

\section{S) ScholaroNE" Manuscript Central}




\title{
The square Thue-Morse tiling for photonic application
}

\author{
LUIGI MORETTI*† and VITO MOCELLA $\dagger \dagger$ \\ $\dagger$ DIMET-University "Mediterranea" of Reggio Calabria, Località Feo di Vito, 89060 Reggio Calabria, Italy
}

$\dagger \dagger$ Institute for Microelectronics and Microsystems of National Council of Research (IMM-CNR), Department of Naples, Via P. Castellino 111, 80131 Naples, Italy

*Corresponding author. Email: luigi.moretti@na.imm.cnr.it

The photonic properties of a two-dimensional photonic aperiodic crystal based on the Thue-Morse substitutional sequence were investigated theoretically. Differently from the traditional photonic quasicrystals based on the Penrose tiling, these structures were obtained by removing the lattice points from a square arrangement, following the inflation rules emerging from the Thue-Morse sequence. The resulting structure does not exhibit the typical translational symmetry of a photonic crystals. In particular, it is well known that the Thue-Morse sequence has a singular continuous Fourier transform. This property was transferred directly on the two-dimensional Thue-Morse photonic aperiodic crystal represented by an array of pillars in air.

The electromagnetic field distribution can be described as a quasi-localized state, with characteristics lying between the localized states, corresponding to the defect state in a photonic crystal, and the Bloch states, as in case of the eigenmode in a photonic crystal. The photonic bandgap formation was explored as a function of pillar radius. Furthermore a preliminary investigation of the defect behaviour in square Thue-Morse tiling was carried out. The electric field in the defect state revealed to be strictly localized in the defect pillar. These structures provide interesting properties which could be used to design novel optical devices.

Keywords: photonic quasicrystal, Thue-Morse sequence, aperiodic order, light propagation;

AMS Subject Classification: 65K05; 90C30 (for example; and not italic)

\section{Introduction}

Photonic crystals are one of the major recent revolutions in optoelectronics. The basic idea proposed by E. Yablonovitch [1] in 1987 was that if one is able to build a material that can forbid the propagation of electromagnetic waves then will be able to control spontaneous emission and to lead interesting quantum electrodynamics effects. The idea proposed for obtaining such photonic bandgaps was based on the use of periodic arrangements of dielectric materials, in analogy to the periodic potential that in solid state physics generates band gaps for electrons in semiconductors. This also appears as a road map to strong photon localization, itself a fascinating but elusive phenomenon [2]. Since these pioneering works, numerous different phenomena and applications have been identified in quantum electronics devices such as distributedfeedback mirror and laser, microwave antennae substrate. Novel exciting phenomena such as superprism, selfguiding, negative refraction are based on the richness of the dispersion relation of photonic crystals. In the electronic semiconductors, only a short-range order is necessary for the formation of an electronic band gap. Amorphous semiconductors exist and have band gaps that are comparable in size to those of crystalline semiconductors. However, electrons form bound states and photons do not. Most of the theoretical demonstration of the existence of an electronic band gap without periodicity is based on simplified tight- 
binding models, which is a reasonable description because electrons can form bound states. Since their discovery [3], the quasicrystals have intrigued scientists from various communities (principally from solidstate physics, but also mathematics and nowadays photonics). Aperiodically driven structures may exhibit a variety of weak forms of rotational symmetries, which are not necessarily bounded by the crystallographic restriction, typical of periodic structures. Following up the solid state physics analogy underlying Yablonovich's idea, some researchers considered aperiodically-ordered systems, i.e. Photonic Quasicrystals (PhQc). Thus, the question about the properties of photonic quasicrystals arose naturally. In 1998, Chan et al. [4] show that an array of pillars arranged on the vertices of an octagonal quasiperiodic tiling exhibits a photonic bandgap. This paper gives the beginning of the investigation of electromagnetic properties of $\mathrm{PhQc}$ (for a review see Refs. 5 and 6). Due to the lack of translational symmetry, they should not have propagation characteristics of Bloch waves, but exhibit unique characteristics of the mixture of light propagation and localization. Some of the photonic bandgaps of a PhQc exhibit a behaviour similar to that typical of a photonic crystal, while others do not. Moreover, it has been noted [4] that one advantage of these aperiodic structures is to present many inequivalent sites and consequently many possible defects. In fact, one fixed set of localized defect modes is produced by removing a pillar from a periodic array. On the other hand, for a quasiperiodic arrangement of pillars, not all of the pillars are equivalent, therefore defect states can be produced with different frequencies and mode patterns. First experimental studies on the emission in aperiodically ordered structures, considering a Penrose-type quasicrystal laser, has been reported in Ref.[7]. In that work, localized modes at variance with extended modes in photonic crystal lasers have been shown. In 2004, Baba's group demonstrated the lasing action in a microcavity composed by a point-defect in the 12-fold symmetric PhQc [8].

The light propagation in a one-dimensional optical multilayer based on an aperiodic substitutional sequence, has been theoretically and experimentally investigated in the last years. The most common examples of one-dimensional aperiodic crystals are the Fibonacci, Thue-Morse (ThMo) and Rudin-Shapiro structures. In particular, ThMo sequence has been extensively studied in mathematical literature, as the prototype of a sequence generated by substitution, with highly non-trivial properties as its Fourier transform being singular continuous. This very peculiar characteristic of the ThMo sequence induces part of the crystallographic community to do not consider ThMo as a crystal due to the lack of a discrete diffraction diagram $[9,10]$. However, it could be interesting to investigate the effects of a purely measure-theoretic property on the propagation, confinement and localization properties of the light.

Several theoretical and experimental works on the light propagation and localization in aperiodic multilayers have been reported in literature [11-14]. The electromagnetic field distribution is uniquely determined by the measure of the energy spectrum. Therefore, since Fibonacci lattices have been demonstrated to have purely singular continuous energy spectrum, the associated electromagnetic states cannot be extended in the Bloch' sense, and can be classified as quasi-localized states, with characteristics lying somewhere between the localized states and Bloch states. Even-Dar Mandel et al. show the probable emergence of extended Bloch wave functions in the electronic energy spectra of the square Fibonacci tiling [15].

Until now, the PhQC investigated are based on the Penrose lattice. Penrose tiles are composed of fat and skinny rhombic tiles and fill the 2D plane nonperiodically. Photonic quasicrystals with high-order symmetry (quasicrystals with six, eight, ten and twelve- fold has been deeply studied in several field of physics) can be obtained by changing the rhombues angle. Recently, in Ref. [16] the authors reported the light propagation and the electric field patterns in a 2D ThMo aperiodic photonic crystal.

In this work the behavior of the photonic bandgap of an array of dielectric pillars based on a 2D ThMo is investigated, with particular attention on the dependence of the photonic bandgap structure on the pillar 
radius. Furthermore, the initial investigation about the defect states behaviour in 2D ThMo structures is reported. The electric field become strictly localized in the defect points of the aperiodic lattice.

\section{The square Thue-Morse tiling}

The 2D ThMo structure investigated here is generated by a direct generalization of the 1D ThMo substitutional rule. The corresponding aperiodic photonic structures are obtained by placing identical dielectric pillars in the lattice points of a square ThMo. Figure 1 shows the first-orders of ThMo square structures: $\mathbf{M}_{1}, \mathbf{M}_{2}, \mathbf{M}_{3}$ and $\mathbf{M}_{4}$, with their complementary structures $\overline{\mathbf{M}}_{i}$. The pillars are assumed to lie in the vacuum, to be dispersionless and lossless, with relative dielectric constant $\varepsilon_{r}=12.25$, which correspond to the silicon in infrared spectrum, and radius $r$. Figure 1 allows noting the inflation rule for $\mathbf{M}_{N}$ that is simply deduced by the inflation rule of the 1D ThMo sequence:

$$
\mathbf{M}_{N+1}=\left|\begin{array}{ll}
\overline{\mathbf{M}}_{N} & \mathbf{M}_{N} \\
\mathbf{M}_{N} & \overline{\mathbf{M}}_{N}
\end{array}\right| \quad \text { and } \quad \overline{\mathbf{M}}_{N}=-\mathbf{M}_{N}
$$

Furthermore, the following general property of the 2D ThMo can be deduced:

$$
R(\pi / 2) \mathbf{M}_{i}= \begin{cases}\mathbf{M}_{i} & \text { for } i \text { even } \\ \overline{\mathbf{M}}_{i} & \text { for } i \text { odd }\end{cases}
$$

where $R(\theta)$ is the rotational matrix of angle $\theta$. Eqs. (1) and (2) lead to infer that $\mathbf{M}_{1}$ can be considered as the basic building block for the 2D ThMo structure. In fact, all of the ThMo structure orders can be obtained by using $\mathbf{M}_{1}$ and $R(\theta / 2) \mathbf{M}_{1}$. These relations are very useful for fabrication purposes, and make the $2 \mathrm{D}$ ThMo photonic crystal more interesting compared to other 2D aperiodic structures.

\section{Light propagation in a defect-free square Thue-Morse tiling}

The photonic bandgap analysis in ThMo structures has been explored comparing two different methods, the Finite Difference Time Domain (FDTD) algorithm used to carry out the density of states (DOS) [17] and the Plane Wave Expansion (PWE) [18] applied to the approximant structure of infinite ThMo structure. The FDTD is an order- $N$ algorithm, where $N$ is proportional to the size of the system, therefore it provides minor efforts from the point of view of computation time. On the other hand the PWE is a $p N$-order where $p$ is the number of calculated bands.

All of the bands at a single $\mathbf{k}$ point have been calculated simultaneously by using the FDTD approach. The bands correspond to the peaks of the system frequency response. The $2 \mathrm{D}$ ThMo structures have been excited with a localized source, such as a narrow Gaussian or delta-function distribution. The large range of spatial frequencies in this source corresponds to a large range of temporal frequencies as the field evolves in time. The actual frequency content that appears is consistent with the applied boundary conditions. A time monitor has been introduced at a point of low symmetry in the 2D ThMo structure to obtain the response and the field amplitude has been monitored as a function of time. The time monitor is converted to a spectral response using a Discrete Fourier Transform operation, at the end of the propagation. The peaks in the spectrum are determined and recorded as the eigenfrequencies of each band. A supercell approximation has been used to overcome the lack of translational symmetry. If one chooses a large supercell, then for each $\mathbf{k}$-point the Fourier-transformed time series will consist of a number of peaks. Adding all contributions from all k's will result in a smooth function of the DOS. The periodic boundary condition is applied to the square supercells. The effect of such 'artificial' condition has been already discussed in Ref. [4]. The authors have shown in Ref [16] that the photonic bandgap structure is already well-defined in the low order structures $\mathbf{M}_{3}$. 
The unit time is represented by $a / \mathrm{c}$, in the FDTD scheme, where $a$ is the minimum distance between two cylinder, and the frequencies are measured in normalised units of $a / \lambda$. The $x y$-plane is discretized into a fine uniform square grid with $40 \times 40$ grid points per basic building block $\left(\mathbf{M}_{1}\right)$. For numerical stability, good convergence and reliability of simulation until frequency of $1 a / \lambda$, we use $3 \times 10^{4}$ time steps, with each time step of the order of $0.025 \mathrm{a} / \mathrm{c}$. The electric field is assumed to be parallel to the axis of the pillars (transverse magnetic mode).

The photonic bandgap formation for different pillar radius $r$, with dielectric constant $\varepsilon_{r}=12.25$, is investigated. Figure 2 reports the behavior of DOS of $\mathbf{M}_{3}$ and a photonic crystal with square arrangement for different radius. The photonic bandgap appears in $\mathbf{M}_{3}$ structures in the same frequency region of the $\mathrm{PhC}$ ones. This effect is due to the 4-fold symmetry of the Fourier transform of $\mathbf{M}_{3}$ structures that can be straightforward recognized from Eq. (1). Therefore the stronger peaks in the Fourier spectrum of ThMo structures in the reciprocal space are in the same position of the reciprocal points of PhC. The presence of bandgap in $\mathrm{PhC}$ is a necessary but not a sufficient condition to have a bandgap in ThMo structures. For example, for $r=0.35 a$ the fourth bandgap of $\mathrm{PhC}$ (at frequency of $0.746 a / \lambda$ ) disappears completely in $\mathbf{M}_{3}$. Each bandgap of $\mathrm{PhC}$ is splitted in ThMo structures. The vanishing of the bandgap in ThMo structures is due to the complex interaction of light with pillars that involves higher-order of Born approximation [19, 20]. The first bandgap (at lower frequencies) is affected by Mie resonances of the single pillar [16, 21]. In fact, as the Mie resonances the position of the first bandgap shifts towards lower frequencies with increasing of pillar radius.

The PWE method has been explored to investigate the band diagram of ThMo structures. $\mathbf{M}_{4}$ has been chosen as approximant structure of 2D ThMo. This structure has 128 "atoms", and provides local structures (hexagon, square, octagon ) that can be replied aperiodically in an infinite ThMo structure. It is important to underline that, differently from the electronic quasicrystals, in case of the PhQc just a lower order of approximant can provide with good approximation the photonic bandgap characteristics. In fact, the potential scattering in our photonic structures that is proportional to the dielectric constant ratio $\left(\varepsilon_{r} / \varepsilon_{a i r}=12.25\right.$ in our case) is higher that the atomic potential in electronic quasicrystals, thus allowing to avoid higher order approximants.

Figure 3 shows the diagram band of $\mathbf{M}_{4}$. The results, in terms of position and width of photonic bandgaps, are in good agreement with DOS plot. Similar results are obtained using the $\mathbf{M}_{3}$ as approximant [15]. Moreover, the bands are very flat $\left(\Delta \omega_{0} / \omega_{0} \cong 10^{-3}\right)$, as shown in Fig.3, thus allowing to deduce that, in case of ThMo structures, a reduction of the group velocity occurs in all directions. In particular the band edge (band I, II, III, and IV) provides the following value of the group velocity: $\mathrm{d} \omega_{\mathrm{I}} / \mathrm{d} k \cong 5 \times 10^{-3} c, \mathrm{~d} \omega_{\text {II }} / \mathrm{d} k \cong 5 \times 10^{-2} c$, $\mathrm{d} \omega_{\text {III }} / \mathrm{d} k \cong 4 \times 10^{-2} c$, and $\mathrm{d} \omega_{\text {IV }} / \mathrm{d} k \cong 2 \times 10^{-2} c$. Therefore using ThMo structures could be possible to design optoelectronic devices based on slow-light effect. The strong interest in slow-light in dielectrics is motivated by the fact that slow light adds functionality to a material by structuring alone. This is of great interest, as it allows to enhance the weak interaction in a material that may be of interest otherwise, such as silicon. Linear effects such as gain, thermo-optic and electro-optic interaction scale with slowdown factor, whereas nonlinear effects may scale with its square [22].

\subsection{Electric field patterns}

The nature of the energy spectrum and its eigenstates are related to the Fourier spectrum. The lack of translational symmetry precludes the presence of the usual extended states observed in photonic crystals. Therefore, the peculiar nature of the critical states have to be considered. Most of them may exhibit quite small group velocities. Generally speaking, critical states exhibit a rather involved oscillatory behaviour, displaying strong spatial fluctuations. The nature of a state is uniquely determined by the measure of the 
spectrum. The singular continuous energy spectrum of ThMo structures provides lattice-like states [23]. In order to investigate the peculiarity of eigenstates of ThMo structures, Fig. 5 reports the electric field patterns in the frequency range between 0.222-0.234 $a / \lambda$ that is between the first two bandgap zones.

The characteristic oscillatory behavior of eigenstates can be noted. The states represented in Fig. 4 (a), (d) and (e) show that the electromagnetic field is localized in the octagonal structures. On the other hand, the states reported in Fig. 4 (b) and (c) show that the maxima and minima of electromagnetic field are in the hexagonal structures. The specific characteristic of the electric field pattern can be exploited for the realization and design of nanocavity arrays based on photonic aperiodic crystal. Optical nanocavities can be used to enhance the spontaneous emission rate as they can dramatically increase electromagnetic DOS with respect to free space. This effect can be used to improve the speed of lasers and to increase the fraction of spontaneously emitted photons that are coupled into a single cavity mode, thereby enabling lasers with low lasing threshold power [24]. In recent years, photonic crystal nanocavities have been extensively studied for spontaneous emission control in order to realize low-threshold laser [25]. Unfortunately, the output power levels of such lasers are extremely low, below the levels needed for many practical applications. To overcome these problems, Altug et al. have been recently proposed two dimensional coupled photonic crystal nanocavity array structures [26]. The possibility to adopt 2D ThMo structures as nanocavity array based on aperiodic crystal is proposed here. Differently from photonic crystal nanocavity arrays, where the resonance of the nanocavity can be achieved for a single frequency, and with a specific electric field pattern, in 2D ThMo a multifrequency nanocavity array exists, and for each frequency the electric field pattern has a different spatial behavior. These characteristics make the ThMo structures very appealing for the design of novel optical devices.

\section{Behavior of defect in square ThMo tiling}

The behavior of defect states in ThMo structures could be more useful than the defect states in photonic crystals. The study of defects in photonic crystals is at least as important as the study of the perfect photonic crystals for many plausible applications in integrated optics. In the ThMo structure arrangement, differently from the periodic arrangement, there is a set of non-equivalent sites, so that defect states with different frequencies and mode patterns can be obtained. For this reason the investigation of defect states in ThMo structures is quite complex and an example of this effect is intended to be provided here. The radius of the pillar, composing the octagonal local structures in $\mathbf{M}_{3}$, is modified (the radius is $0.35 a, \varepsilon_{\mathrm{r}}=12.25$ ) while the defect radius, $r_{d}$ is variable. The $\mathbf{M d}_{3}$ (Fig. 5(a)) can be adopted as a basic building block to obtain higher order ThMo, following the Eqs. (1) and (2). Therefore, defected ThMo structures are obtained because generated by a defected basic building block. The DOS of $\mathbf{M d}_{3}$ for different defect radius from $0.2 a$ to $0.5 a$ has been reported in fig. 5(b). Comparing the DOS of the defect free structures $\left(r_{d}=0.35 a\right.$, in Fig. 5) to the defected structures, some defect peaks appear due to the presence of the defect states. In particular, for $r=0.5 a$ some defect states appear in each photonic bandgaps of ThMo. Fig. 6 reports the electric field patterns of the defect state for $r_{d}=0.5 a$, at a frequency of 0.207 and $0.241 a / \lambda$ (Figs. 6 (a) and (b), respectively) and at a frequency of $0.361 \mathrm{a} / \lambda$ (Fig. 6 (c)). The electric field is strongly localized in the defect pillar as a dipole mode.

\section{Conclusions}

The photonic properties of a square ThMo structure were investigated in this work. The DOS for different pillar radius was calculated and an analysis of bandgap formation was reported. The electric field patterns show an oscillator behaviour due to the lack of both extension and localization of the ThMo states. This 
characteristic, jointly with the low group velocity of light in all the directions, can be exploited to realize arrays of nanocavities.

Moreover a preliminary study of the defect behaviour in ThMo structures was reported. The defect states are localized in the defect point. These structures show interesting characteristics for the design of novel optical devices.

\section{References}

[1] E. Yablonovich, Phys. Rev. Lett. 58, 2059 (1987)

[2] S. John, Phys. Rev. Lett. 58, 2486 (1987).

[3] D. Shechtman et al., Phys. Rev. Lett. 53, 1951 (1984).

[4] T. S. Chan, C. T. Chan, and Z. Y. Liu, Phys. Rev. Lett. 80, 956 (1998).

[5] E. Macià, Rep. Prog. Phys. 69, 397 (2006).

[6] W. Steurer and D. Sutter-Widmer, J. Phys. D: Appl. Phys. 40, R229 (2007).

[7] M. Notomi et al., Phys. Rev. Lett. 92, 123906 (2004).

[8] K. Nozaki and T. Baba, Appl. Phys. Lett. 84, 4875 (2004).

[9] R. Lifshitz, Fund. Phys. 33, 1703 (2004).

[10] Discussions on “What is a crystal?”, Z. Kristallog. 222, 308-319 (2007).

[11] X. Jiang et al, Appl. Phys. Lett. 86, 201110 (2005).

[12] L. Dal Negro et al, Appl. Phys. Lett. 84, 5186 (2004).

[13] L. Dal Negro et al, Appl. Phys. Lett. 86, 261905 (2005).

[14] L. Moretti et al, Opt. Express 14, 6264 (2006)

[15] S. Even-Dar Mandel and R. Lifshitz Phil. Mag. 86, 759 (2006).

[16] L. Moretti and V. Mocella, Opt. Express 15, 15314 (2007)

[17] C. T. Chan et al, Phys. Rev. B 51, 16635 (1995).

[18] S. Johnson and J. Joannopoulos, Opt. Express 8, 173 (2001).

[19] A. Della Villa et al., Phys. Rev. Lett. 94, 183903 (2005). 
[20] M. Born and E. Wolf, Principles of Optics (Cambridge University Press) $7^{\text {th }}$ edition.

[21] C. Rockstuhl el al., Opt. Lett. 31, 1741 (2006).

[22] T. F. Kraus, J. Phys. D: Appl. Phys. 40, 2666 (2007).

[23] C. S. Ryu el al, Phys. Rev. B 46, 5162 (1992)

[24] Y. Yamamoto et al, Phys. Rev. B 44, 657 (1991).

[25] H. G. Park el al, Science 305, 1444 (2004).

[26] H. Altug and J. Vučkovic, Appl. Phys. Lett. 84, 161 (2004). 


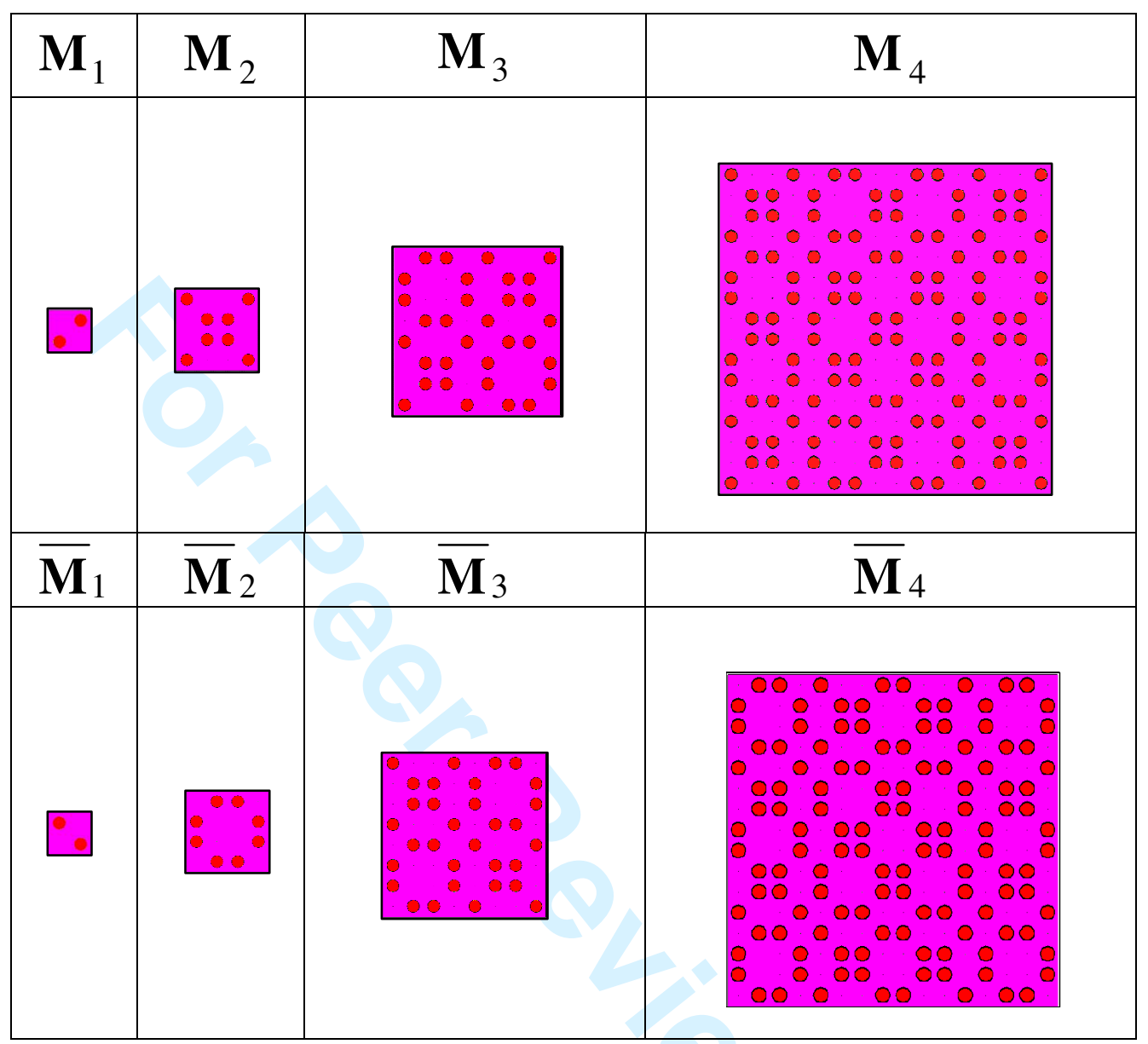

Fig. 1 First orders and their complements of square Thue-Morse photonic aperiodic crystal made of dielectric cylinders lying in the vacuum. The cylinders are located at the vertices of a two dimensional square ThueMorse structure. 


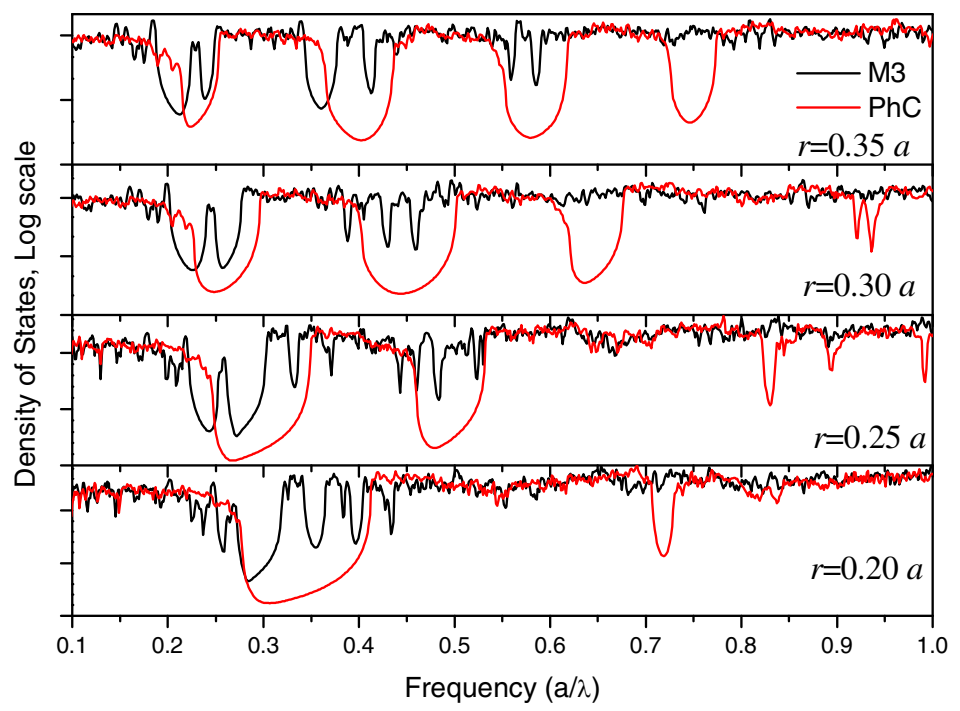

Fig. 2 Density of States of $\mathbf{M}_{3}$ ThMo structures for different cylinder radius. The DOS behavior is compared with that of the corresponding perfect square photonic crystal. 


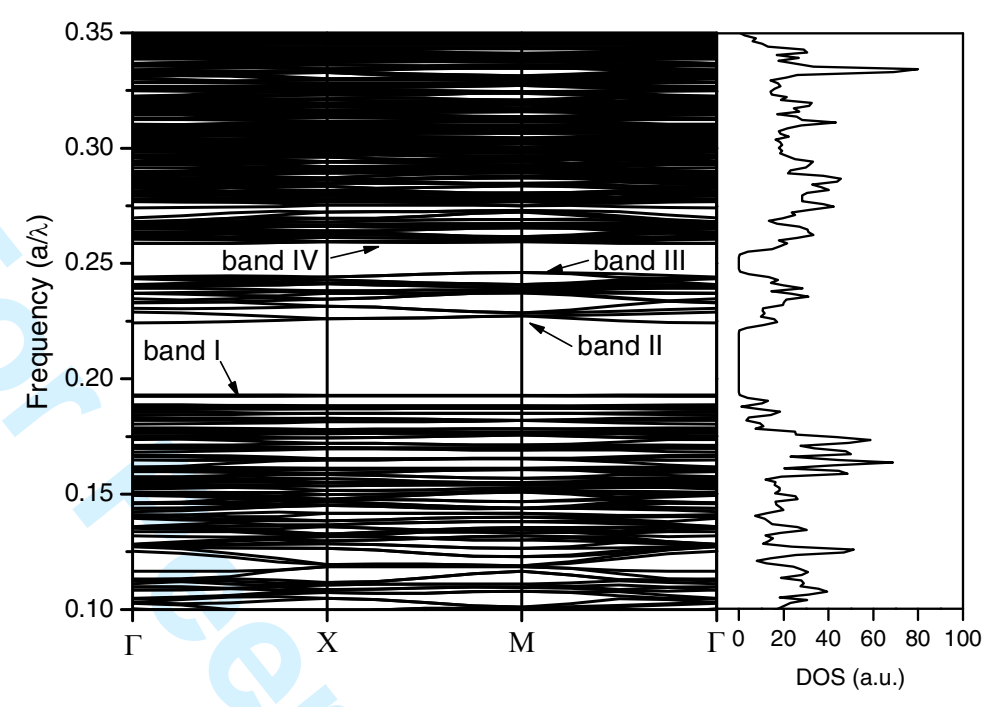

Fig. 3 Band diagram of $\mathbf{M}_{4}$ ThMo approximant with $r=0.35 a$ compared with the DOS. 


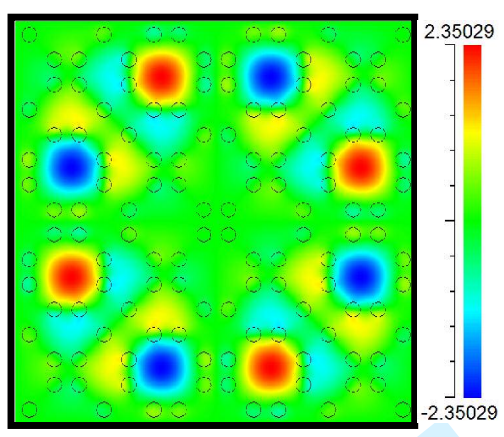

(a)

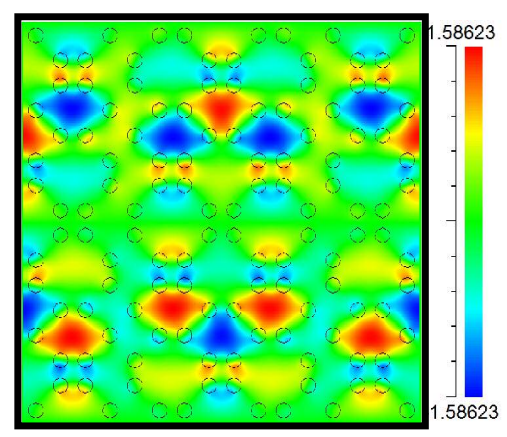

(b)

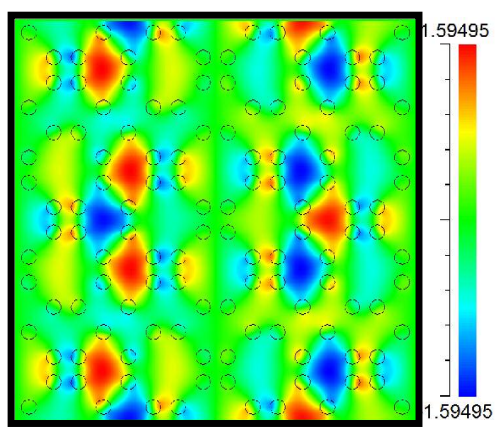

(c)

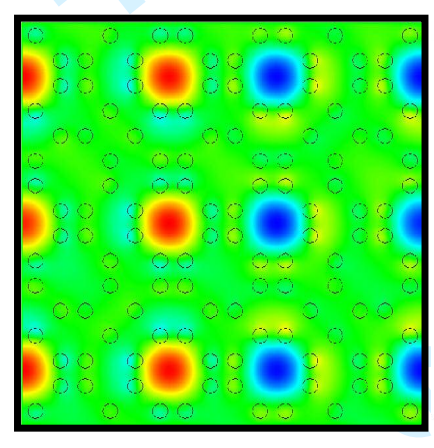

(d)

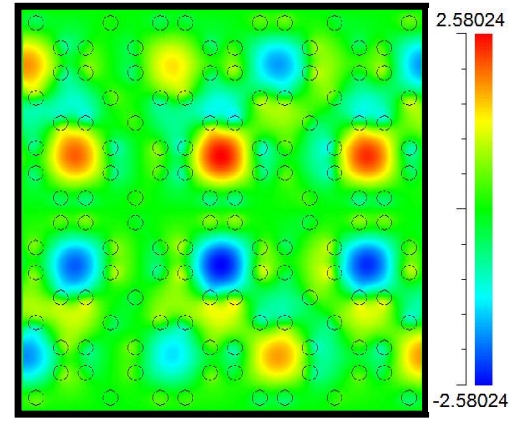

(e)

Fig. 4: Electric field patterns: a) $\Gamma$-point at $0.241 \mathrm{a} / \lambda$; b) and c) $\Gamma$-point at 0.258 and $0.259 a / \lambda$; d) M-point at $0.246 a / \lambda$; e) X-point at $0.244 a / \lambda$. 

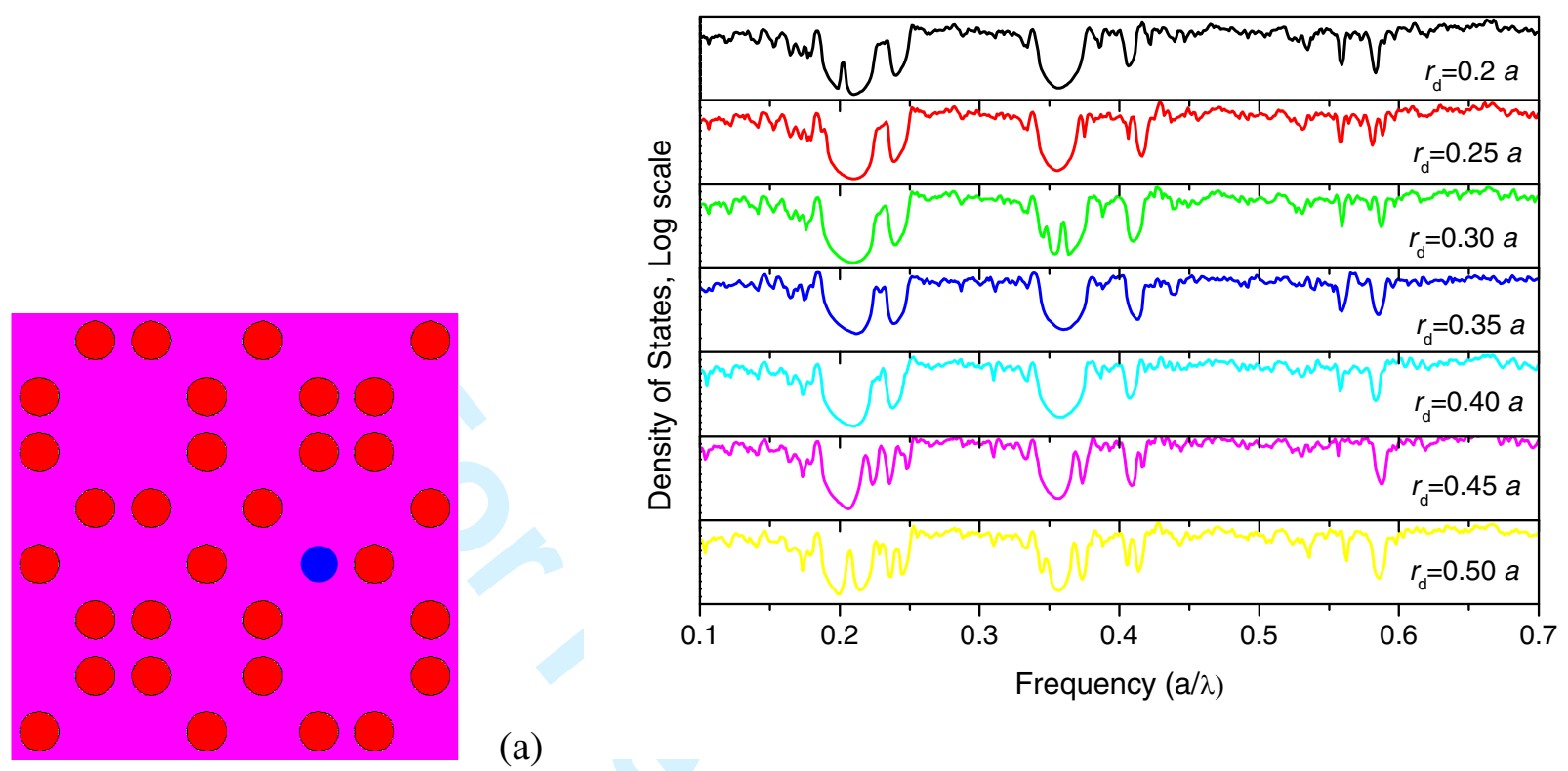

(a)

Fig. 5 (a): ThMo defected structure $\mathbf{M} \mathbf{d}_{3}$, the defect has been induced changing the radius $r_{d}$ of blue cylinder; (b) DOS for different size of cylinder defect. 

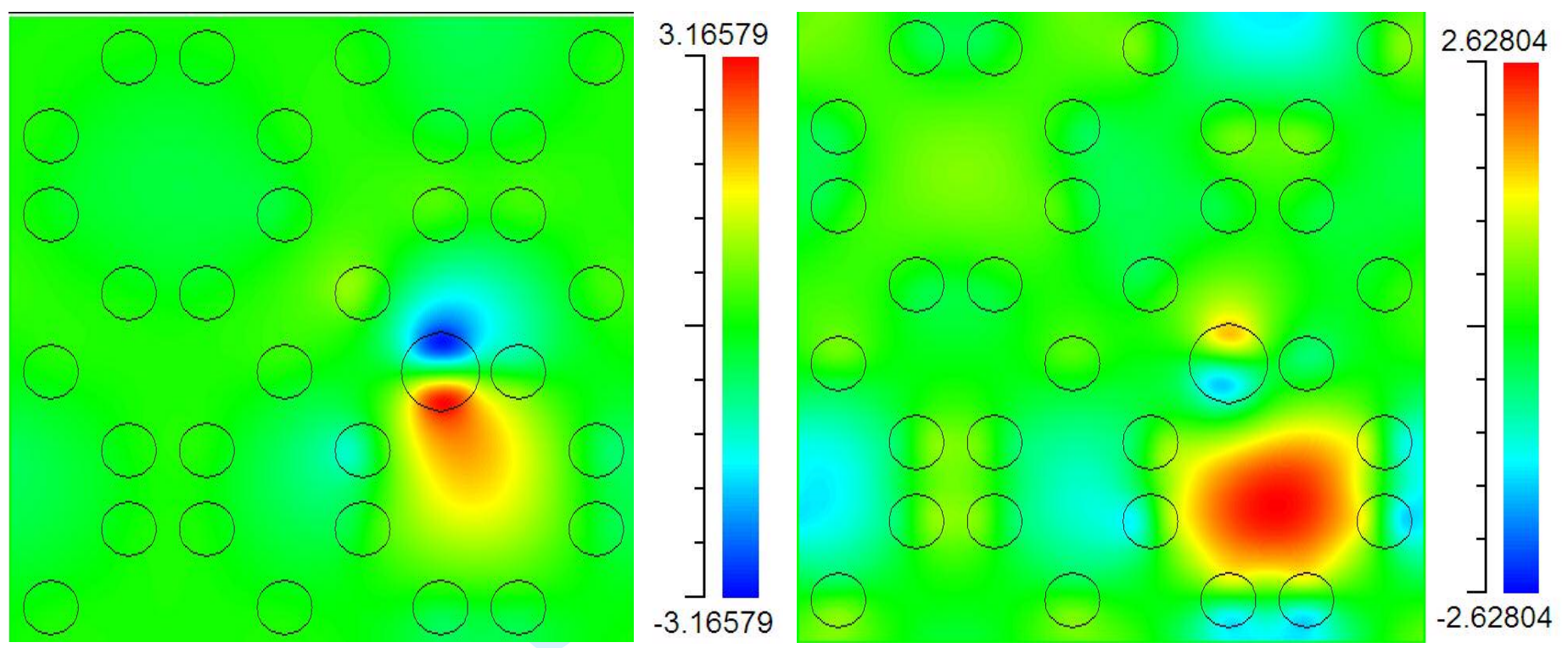

a)
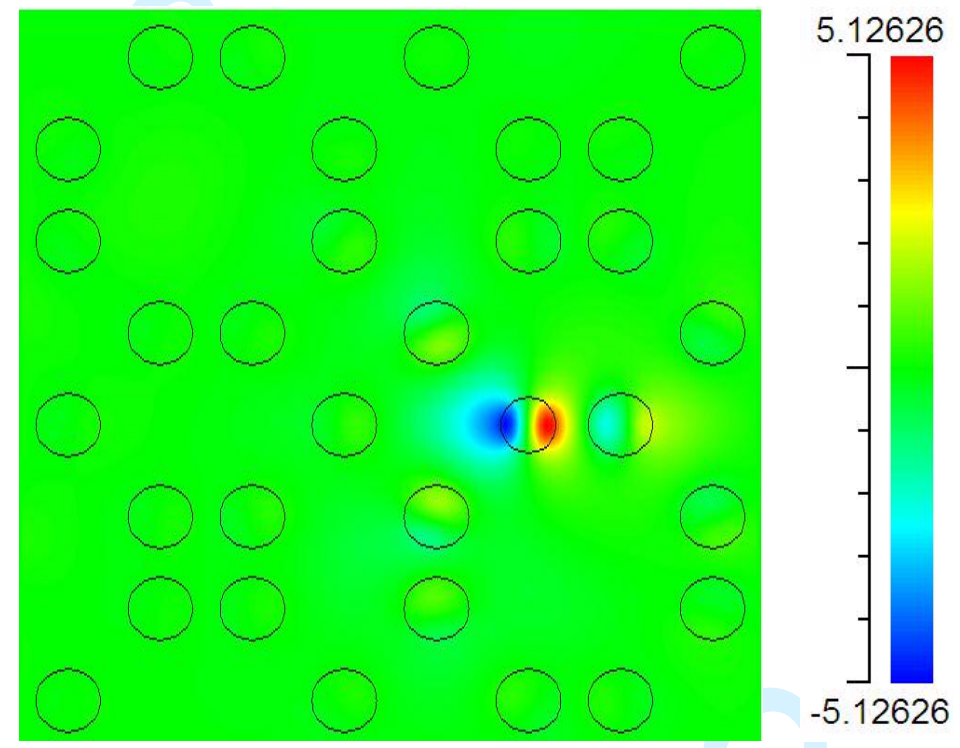

b)

c)

Fig. 6 Electric field patterns at frequency of $0.207 a / \lambda$ (a), 0.241 (b) for $r_{d}=0.5 a$, and $0.361 a / \lambda$ (c) for $r_{d}=0.3$ $a$. 